\section{Successful surgical treatment of intractable post-radiation rectal bleeding}

\author{
Rezvan Mirzaei, ${ }^{1}$ Bahar Mahjoubi, ${ }^{1}$ \\ Jalil Shoa, ${ }^{2}$ Roozbeh Cheraghali, ${ }^{3}$ \\ Zahra Omrani ${ }^{1}$
}

${ }^{1}$ Colorectal Research Center, Iran University of Medical Sciences, Tehran; ${ }^{2}$ Gilan University of Medical Sciences, Rasht; ${ }^{3}$ Vascular \&Endovascular Surgery, Golestan University of medical Sciences, Gorgan, Iran

\begin{abstract}
Patients will typically present symptoms of chronic post-radiation colitis and proctitis 8-12 months after finishing their treatment. Endoscopic methods play the main role the treatment of bleeding caused by post-radiation colitis and proctitis. Surgical treatment is required for remained approximately $10 \%$ of patients. Here we present a 64 year old female with metastatic breast cancer, who was referred to us for intractable rectal bleeding. Total colonoscopy and rigid rectosigmoidoscopy revealed proctitis, rectal and sigmoidal telangiectasis, multiple necrotic ulcers between 15 to $30 \mathrm{~cm}$ from the anal verge, and also huge ishemic ulcer with patchy necrotic areas about $10 \mathrm{~cm}$ from the anal verge. This abnormal irradiated part was resected and then mucosectomy of the remnant rectum, both transabdominally and transanally was done. We performed pullthrough technique of normal proximal colon to anal region through the remnant rectal wall and finally did coloanal anastomosis. Diverting stoma was not made because of anastomosis in anal region. With this technique we can achieve benefits such as avoidance of harsh dissection in a frozen pelvis and its consequences, we can avoid intra-abdominal anastomosis, there is no need to a diverting stoma and, most important of all, definite bleeding control.
\end{abstract}

\section{Introduction}

Most large series estimate the incidence of chronic radiation injury to be between $5 \%$ to $11 \%{ }^{1}$ Patients will typically present symptoms of chronic post-radiation colitis and proctitis 8-12 months after finishing their treatment. ${ }^{1,2}$ The pathophysiology of these complications is related to ischemic injury. ${ }^{1,2}$ Diagnosis is usually done with endoscopy. Main treatment in this condition is Sucralfate enemas, Asacol, corticosteroid, and short chain fatty acids are also used. Endoscopic methods play the main role in treatment of bleeding caused by post-radiation colitis and proctitis. Argon Plasma Coagulation (APC) is routinely used. Remission rate with this method is 80 $90 \%{ }^{1-4}$ Surgical treatment is required for approximately $10 \%$ of patients, whose symptoms cannot be managed with medical or endoscopic therapy. Surgery faces several major challenges in postradiation colitis and proctitis. Irradiation of bowels has more risk of anastomosis leakage. Also dissection in radiated pelvis is difficult. One of surgical options is bowel diversion, but its effect on bleeding is uncertain. ${ }^{3,4}$ Amount of data and solutions presented by surgeons is low and limited to case presentations. Here we present a 64 year old female with metastatic breast cancer, who was referred to us for intractable rectal bleeding after radiation therapy.

\section{Case presentation}

A 64 year old female, who underwent left mastectomy due to breast cancer 2 years ago, received 8 chemotherapy sessions and 48 pelvic radiation courses because of spinal metastasis. She didn't have any other disease in her medical history. Five months before consultation she encountered notable rectal bleeding and smelly diarrhea. The patient had two colonoscopic studies that both show evidence of inflammation, telangiectasis of the rectum and sigmoid, and reported a large necrotic ulcer about 10 $\mathrm{cm}$ from the anal verge. Bleeding episodes had an increasing pattern in their volume and frequency, so that she was admitted in other emergency departments two times for blood transfusion. Despite repeated blood transfusion she was referred to us when her hemoglobin was $6 \mathrm{mg} / \mathrm{dL}$. She complained of fecal frequency and diarrhea with severe mal odor of her fecal material. Patient underwent total colonoscopy and rigid rectosigmoidoscopy again and they revealed proctitis, rectal and sigmoidal telangiectasis, multiple necrotic ulcers between 15 to $30 \mathrm{~cm}$ from the anal verge, and also huge ischemic ulcer with patchy necrotic areas (Figure 1) about $10 \mathrm{~cm}$ from anal verge. She had been received medical treatment by Sucralfate, Asacol, and Hydrocortisone enemas and 3 sessions of endoscopic intervention by APC. Investigations revealed she had the good cardiac and respiratory conditions.

According to the severity of bleeding, failure of medical and endoscopic treatments, and also mucosal necrosis that caused her smelly diarrhea, the surgical
Correspondence: Roozbeh Cheraghali, Vascular \&Endovascular Surgery, Golestan University of medical Sciences, Sina Hospital, Hasan Abad Sq,Tehran,Iran, postal code 1136746911, Iran

Tel:+98.21.66348564; Fax:+98.2166348564 E-mail: Dr.r.Cheraghali@goums.ac.ir, roozbehcheraghali81@gmail.com

Key words: Radiation; proctitis; rectal bleeding; surgery.

Contributions: All authors contributed to the study conception and design. Material preparation, data collection and analysis were performed by EM, RC, ZO, BM and JS. The first draft of the manuscript was written by $\mathrm{ZO}$ and all authors commented on previous versions of the manuscript. All authors read and approved the final manuscript.

Conflict of interest: The authors have $\mathrm{n}$ conflict of interest to declare.

Availability of data and materials: Data of patient are available at the Colorectal Research Center of Rasool, Akram Hospital, Tehran, Iran

Ethics approval and consent to participate: No ethical committee approval was required for this case report by the hospital, because this article does not contain any studies with human participants or animals. Informed consent was obtained from the patient included in this study.

Consent for publication: The patient gave her written consent to use her personal data for the publication of this case report and any accompanying images.

Received for publication: 7 March 2021. Revision received: 29 December 2021.

Accepted for publication: 3 January 2022.

This work is licensed under a Creative Commons Attribution NonCommercial 4.0 License (CC BY-NC 4.0).

(C) Copyright: the Author(s), 2021

Licensee PAGEPress, Italy

Surgical Techniques Development 2021; 10:9125

doi:10.4081/std.2021.9125

intervention seemed inevitable. Followed by the presentation of the case in a multidisciplinary session and according to consultations, she was prepared for the operation. During laparotomy, we encountered a frozen pelvis and there was a small perforation site on the anterior wall of the rectum that had been sealed by uterus. To avoid wide pelvic exenteration, after releasing the upper rectum and sigmoid, we resected this abnormal irradiated part and then accomplished mucosectomy of the remnant rec- 
tum, both transabdominally and transanally. With releasing of splenic flexure, we performed pull-through technique of normal proximal colon to the anal region through the remnant rectal wall and finally did coloanal anastomosis. Diverting stoma was not made because of anastomosis in the anal region. The operation finished with insertion of a drain above pelvic area. Patient spent post-operation period without any problem and at 2 years follow up she had a favorable situation.

\section{Discussion}

Failure of medical and endoscopic treatments in post-radiation colitis and proctitis is rare and may present surgeons with major challenges. In bleeding cases, diversion has no clear benefit and also it has no efficacy in necrosis and perforations. Postradiation proctitis may be a sign that alerts us of having different severity of post-radiation problems in other pelvic organs.

Do et al. in 2017 argued treatment options in post-radiation proctitis. They recommended two surgical options in those

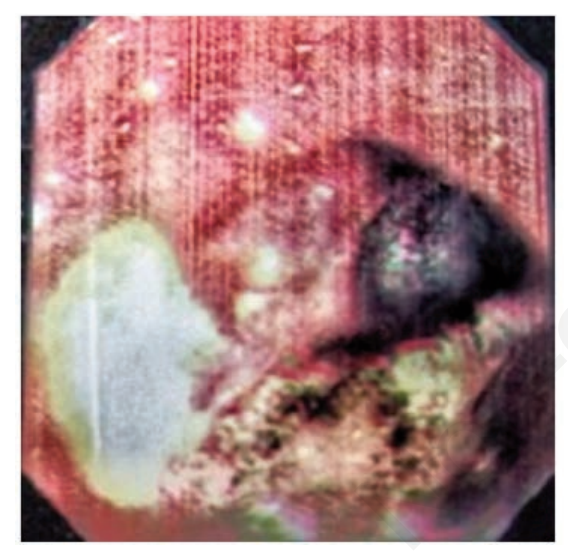

Figure 1. Necrotic rectal ulcer in our patient in colonoscopy. who did not respond to medical and endoscopic medications. First, proctectomy with anastomosis and diversion stoma. Second, Abdominoperineal Resection (APR) with permanent stoma. ${ }^{5}$ Grodskey et al. in 2015 discussed post-radiation proctopathy. ${ }^{6}$ The operative options they mentioned were similar to Do et al. Authors recommended proctectomy with anastomosis and diverting stoma in patients who received less than 4000-4500cGy total dose of radiation. Another surgical option that they considered for other patients is mucosal proctectomy with sleeve coloanal anastomosis, between the non-radiated proximal colon and anal canal. Jensen et al. in 2003 argued surgical strategies in these patients. They recommended resection and coloanal anastomosis with or without colonic J-Pouch. ${ }^{7}$ Between older articles, Lucarotti et al. in 1991 discussed this subject. In their paper, coloanal anastomosis with J-Pouch was recommended, and they urged to avoid coloanal sleeve anastomosis. ${ }^{8}$

If we encountered a post-radiation frozen pelvis in a patient such as ours, we should decide and think about what price we have to pay if we start pelvic exenteration, and whether it is reasonable or not. We agree that in a condition of bleeding, extensive necrosis and perforation, should do a resection of the involved bowel to eliminate the source of problem, but we should alter our resection technique according to the condition of bowel, pelvis and patient. With this technique we can achieve benefits such as avoidance of harsh dissection in a frozen pelvis and its consequences, intra-abdominal anastomosis, and there is no need for a diverting stoma. Most important of all, it is a definite bleeding control method. It seems that our technique can be an effective option in those rare and critical patients.

\section{Conclusions}

Mucosectomy of distal rectum, pull intact proximal colon through the remnant rectal wall (pull through technique) and then coloanal anastomosis is an appropriate option in post radiation frozen pelvis patients.

\section{References}

1. Nivatvongs S. Radiation injuries to the small and large intestine. In: Gordon $\mathrm{PH}$, Nivatvongs $\mathrm{S}$ editors. Principles and practice of surgery for the colon, rectum, and anus. 3rd ed. NewYork: Informa Healthcare USA: 2007. p.1015-1028.

2. Raiche I, Moloo H. Radiation, microscopic, ischemic colitis. In: Steele SR, Hull TL, Reed TE, et al. (eds.) The ASCRS textbook of colon and rectum surgery. 3rd ed. New York: Springer: 2016. p.951-965.

3. Henson C. Chronic radiation proctitis: issues surrounding delayed bowel dysfunction postpelvic radiotherapy and an update on medical treatment. Therap Adv Gastroenterol 2010;3:359-65.

4. Takemoto S, Shibamoto Y, Ayakawa S, et al. Treatment and prognosis of patients with late rectal bleeding after intensity-modulated radiation therapy for prostate cancer. Radiation Oncol 2012;7:87

5. Do NL, Nagle D, Poylin VY. Radiation proctitis: current strategies in management. Gastroenterol Res Pract 2011;2011:917941.

6. Grodsky MB, Sidani SM. Radiation proctopathy. Clin Colon Rectal Surg 2015;28:103-11.

7. Jensen MH, Wang J, Denham JW. Bowel injuries: current and evolving management strategies. Seminars Radiation Oncol. 2003;13:357-371.

8. Lucarotti ME, Mountford RA, Bartolo DC. Surgical management of intestinal radiation injury. Dis Colon Rectum 1991;34:865-9. 